\title{
Individual position diversity in dependence socioeconomic networks increases economic output
}

\author{
Wen-Jie Xie ${ }^{1,2}$, Yan-Hong Yang ${ }^{1,2}$, Ming-Xia Li ${ }^{2,3}$, Zhi-Qiang Jiang ${ }^{1,2}$ and Wei-Xing Zhou ${ }^{1,2,4^{*}}$ (1)
}

"Correspondence:

wxzhou@ecust.edu.cn

'School of Business, East China

University of Science and

Technology, Meilong Road, 200237,

Shanghai, China

${ }^{2}$ Research Center for Econophysics,

East China University of Science and

Technology, Meilong Road,

Shanghai, China

Full list of author information is

available at the end of the article

\section{Springer}

\begin{abstract}
The availability of big data recorded from massively multiplayer online role-playing games (MMORPGs) allows us to gain a deeper understanding of the potential connection between individuals' network positions and their economic outputs. We use a statistical filtering method to construct dependence networks from weighted friendship networks of individuals. We investigate the 30 distinct motif positions in the 13 directed triadic motifs which represent microscopic dependences among individuals. Based on the structural similarity of motif positions, we further classify individuals into different groups. The node position diversity of individuals is found to be positively correlated with their economic outputs. We also find that the economic outputs of leaf nodes are significantly lower than that of the other nodes in the same motif. Our findings shed light on understanding the influence of network structure on economic activities and outputs in socioeconomic systems.
\end{abstract}

Keywords: position diversity; socioeconomic network; network motif; economic output

\section{Introduction}

Considerable studies have offered us a deep understanding of the influence of network structures on the dynamics of complex systems, such as the spreading of diseases and information [1] and emerging of collaborations [2]. However, the connection between network position and economic output is less studied. It is reported that specific network structures may enhance economic outputs [3-7]. Eagle et al. argue that the diversity of individual relationships within a community strongly correlates with economic development of communities [5]. Furthermore, Bettencourt et al. and Ortman et al. find that the diversity of relationships is positively correlated with the productivity of individuals and communities [6,7].

Network motifs are building blocks of complex networks [8-10]. It is found that network motifs of social networks may reflect the driving forces for forming social structures [11, 12]. Similar to the friendship networks of US students [13], Xie et al. studied triadic motifs in dependence networks of virtual societies and found that low level individuals have preference of forming links to high level individuals [4]. Their findings in virtual world are consistent with empirical findings in real society that 'collaboration is easier when both

(c) The Author(s) 2017. This article is distributed under the terms of the Creative Commons Attribution 4.0 International License (http://creativecommons.org/licenses/by/4.0/), which permits unrestricted use, distribution, and reproduction in any medium, provided you give appropriate credit to the original author(s) and the source, provide a link to the Creative Commons license, and indicate if changes were made. 
partners share the same social status, and the probability of partnership formation decreases significantly as the status gap between the partners increases' [3].

Understanding the structure and function of social networks are of great importance to investigate economic activities and outputs in social systems [11, 14-19]. In real world, social networks are not large and samples are biased [13, 20, 21], which hinders the empirical investigation of relationship between network structures and economic activities in social system. Some other empirical studies have shown that individuals' or firms' network positions are closely related to success measured in terms of economic production [22-25]. In the era of big data, information technology provides us alternative methods to collect data of social relationships and economic activities, for example, massively multiplayer online role-playing games (MMORPGs) [26-30], which enable us to study complex social and economic behaviours of human in online social systems [31-33]. Bainbridge et al. also emphasized the scientific potential of virtual world for future research [31]. Empirical studies show that social behaviours in MMORPGs are representative of human behaviours in many aspects in real society [12, 34-39].

In this paper, we construct dependence networks based on weighted friendship networks of individuals and identify 30 distinct motif positions in 13 directed triadic motifs which represent local dependence among individuals. Using the $k$-means algorithm, we further classify individuals into $k$ clusters based on the motif position profiles. Our results indicate that the motif position of individuals do have great influence on their economic outputs.

\section{Materials and methods}

\subsection{Data description}

We use a huge database recorded from 124 servers to investigate the potential connection between network structure and economic output for individuals within the virtual world of a popular Massively Multiplayer Online Role-Playing Game (MMORPG) in China. There are two opposing camps or societies in a virtual world residing in a server, thus giving us 248 virtual societies. There exist great differences about the numbers of avatars among different virtual societies. The populations of virtual worlds vary from thousands up to fifty thousand. The distribution of the number of avatars in each virtual world is drawn in Figure 1(G). In each society, three professions have different skills. The advantage of warrior's skill is the power of attack, the advantage of mage's skill is the power of defense, and the advantage of priest's skill is the ability to cure illness. An avatar can be a warrior, a priest, or a mage. To improve their skills, the avatars cooperate with friends to accomplish tasks. The more friends, the higher efficiency. Two individuals $i$ and $m$ are allowed to establish social ties to satisfy their desire of making friends and enhance their utility of collaborations. The strength of social ties is measured by the intimacy $I_{i, m}$, which increases according to the collaborative activities of $i$ and $m$ if they belong to the same society; otherwise, $I_{i, m}$ remains zero if $i$ and $m$ belong to two different societies. Hence the friendship networks of the two camps are essentially separated. As a measure of closeness to each friendship, the values of intimacy are recorded every day. When two individuals in the same society form a team and collaborate to accomplish a task, their intimacy increases. The evolving intimacy allows us to track the evolution of the cooperation behavior in the socioeconomic networks. Each individual can maintain a friendship list, denote as $\mathcal{F}_{i}$ for individual $i$. The social tie is symmetric: if $i \in \mathcal{F}_{m}$, then $m \in \mathcal{F}_{i}$. In addition to the 


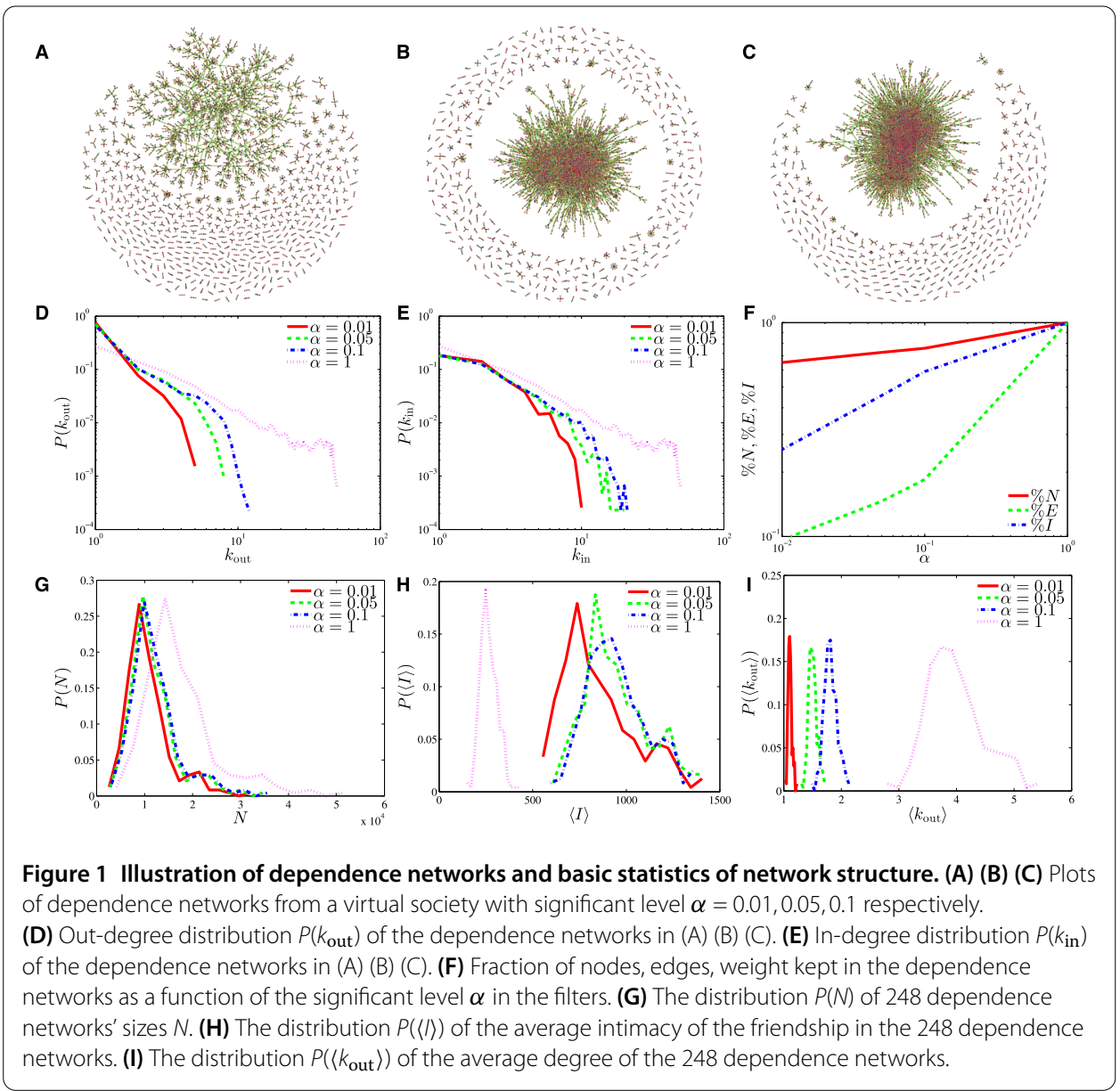

friendship network, the game data contains other socioeconomic networks, such as the face to face trading networks between initiators and receivers, the vendor trading networks between vendors and costumers, the mail networks between senders and receivers, the mentor networks between students and mentors, the kill networks between killers and victims. In this paper, our focus is the friendship network. More details of the database can be found in our earlier works about the triadic motifs in dependence networks [4] and skill complementarity in collaboration networks [40].

\subsection{Economic outputs of individuals}

We measure the economic output by converting the virtual money and items into a standardized currency for each individual. There are two virtual currencies, Xingbi and Jinbi. The Xingbi and Jinbi can be exchanged in the built-in platform in each virtual world. The virtual system has an approximately stable exchange rate between Xingbi and the Chinese currency Renminbi. Jinbi is produced by the economic activities of the individuals when they form a team and collaborate to accomplish the tasks. The currency Jinbi and virtual items, such as weapons, clothes, and medicines, are awarded to individuals when monsters are killed and tasks are accomplished.

We convert the produced items and Jinbi to Xingbi to obtain the real economic output for each individual on each day. On average, the normal life span of virtual societies is 
close to 5 months [40]. Therefore, we calculate the output of each individual for a fixed period of 145 days for all virtual societies, denoted as $y_{i}=\ln \sum_{t=1}^{t=145} y_{i, t}$.

\subsection{Construction of dependence network}

For a given friendship network, we construct a dependence network by removing the insignificant edges based on statistical validation [41]. First, we define the relative intimacy of individual $m$ in reference to all friends of individual $i$ as $w_{i, m}=I_{i, m} / \sum_{m=1}^{N} I_{i, m}$. Obviously, $w_{i, m} \neq w_{m, i}$. Following the statistical validation [41], a directed tie $i \rightarrow m$ is significant at the level of $\alpha$ if

$$
\alpha_{i, m}=1-\left(k_{i}-1\right) \int_{0}^{w_{i, m}}(1-x)^{k_{i}-2} \mathrm{~d} x<\alpha
$$

where $k_{i}$ is the degree of individual $i$. If $\alpha_{m, i}<\alpha$, the directed tie $m \rightarrow i$ is significant. For each society, we set a significant level $\alpha$ and remove those insignificant links, resulting a directed dependence network. The disparity filter is applied to each node. The intimacy of all links of node $i$ are normalized and only the links remain for which equation (1) holds. If link $i \rightarrow m$ is significant, it means that individual $m$ is relatively important to individual $i$ in $i$ 's friends. In other words, individual $i$ depends on individual $m$. By the disparity filter [41], the dependence networks are the backbones of the original friendship networks.

Figure 1(A), (B), (C) show the topological structure of the dependence network constructed from a virtual society with significant level $\alpha=0.01,0.05,0.1$, respectively. We analyze the out-degree distribution $P\left(k_{\text {out }}\right)$ and in-degree distribution $P\left(k_{\text {in }}\right)$ of dependence networks for different significant level $\alpha=0.01,0.05,0.1$ in Figure 1(D), (E). The degree distribution of the dependence network is obviously different from the original friendship network $(\alpha=1)$. Using a smaller significance level $\alpha$, the disparity filter reduces more edges. For different significant level $\alpha$, the fractions of nodes, edges, weight kept in the dependence network increase with the significant level $\alpha$ as shown in Figure 1(F). And the average degree of the 248 dependence networks is monotone increasing with the significant level $\alpha$ in Figure 1(I). For different significant levels $\alpha=0.01,0.05,0.1$, the distributions $P(N)$ of the 248 dependence networks' sizes $N$ have nearly the same shape in Figure 1(G). The numbers of the avatars range from one thousand to fifty thousand. Comparing with the global threshold filter, the disparity filter considers the relevant edges and ensures that the edges with small intimacy are not neglected. So we can find that the average intimacy kept in the dependence network is not monotone decreasing with the significant lever $\alpha$ in the Figure 1(H).

\subsection{Quantifying position ratio profile}

Following the method [9] to identify motifs, we are able to identify 13 different directed triadic motifs, as shown in Figure 2, in dependence networks. These motifs uncover the dependence structures among individuals at the microscopic level. For example, motif (2) $\leftarrow$ (1) $\rightarrow$ (2) stands for the situation that one individual depends on the other two individuals, motif (3) $\rightarrow$ (4) $\rightarrow$ (5) means that one individual depends on another individual which in turn depends on the third individual, whereas motif $(a) \rightarrow(b) \rightarrow(c) \rightarrow$ (a) represents the situation that individual $a$ depends on individual $b$, individual $b$ depends on individual $c$, and individual $c$ also depends on individual $a$. We can further locate 30 distinct motif positions [42] within the 13 different directed triadic motifs, as shown in Figure 2. We directly 
Figure 2 Plots of 30 unique positions $j \in\{1,2, \ldots$, $30\}$ in 13 directed triadic motifs.

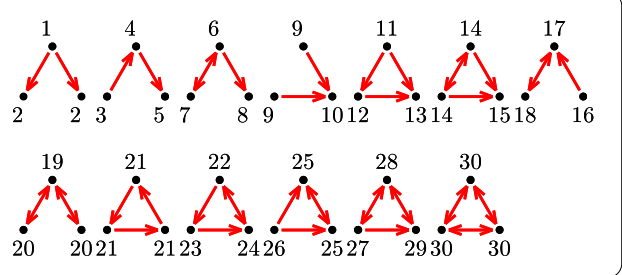

enumerate the relative frequency $p_{i, j}$ that individual $i$ appears in position $j$ across the 13 motifs, which gives the motif position ratio profile $p_{i}=\left(p_{i, 1}, p_{i, 2}, \ldots, p_{i, 30}\right)$ for individual $i$. Note that we have $\sum_{j=1}^{30} p_{i, j}=1$.

Hence, we first define the $z$-sore of occurrence frequency for position $j$ :

$$
Z_{i, j}=\frac{p_{i, j}-\left\langle p_{i, j}\right\rangle}{\sigma\left(p_{i, j}\right)}
$$

where $\left\langle p_{i, j}\right\rangle=\sum_{i=1}^{N} p_{i, j} / N$ and $\sigma\left(p_{i, j}\right)$ are the mean and standard deviation of $p_{i, j}$ over all individuals in the dependence network of a given virtual society $[8,10]$. The structural similarity between individual $i$ and $m$ is thus defined as the correlation coefficients between $Z_{i}$ and $Z_{m}$, such that,

$$
s_{i, m}=\frac{E\left[\left(Z_{i, j}-\left\langle Z_{i}\right\rangle\right)\left(Z_{m, j}-\left\langle Z_{m}\right\rangle\right)\right]}{E\left[\left(Z_{i, j}-\left\langle Z_{i}\right\rangle\right)^{2}\right]^{1 / 2} E\left[\left(Z_{m, j}-\left\langle Z_{m}\right\rangle\right)^{2}\right]^{1 / 2}},
$$

where $E[x]$ is the mathematical expectation of $x$.

\subsection{Classifying individuals based on their position ratio profile}

We employ the $k$-means algorithm to classify $N$ individuals based on their position ratio profiles $\left\{Z_{i}\right\}$ in the dependence social networks. The position ratio profile of individual $i$ is written as $Z_{i}=\left(Z_{i, 1}, Z_{i, 2}, \ldots, Z_{i, 30}\right)$. For each virtual society, we can get a matrix of position ratio profile, denoted by $\mathcal{Z}$, in which rows correspond to individuals and columns correspond to positions in triadic motifs. By adopting the $k$-means algorithm, we partition the individuals into $k$ clusters in terms of minimizing the sum, over all clusters, of the within-cluster sums of point-to-cluster-centroid distances. The partition algorithm is implemented in an iterative way. To evaluate the optimal number of clusters, we calculate the clustering evaluation object containing Davies-Bouldin index values and find the minimum criterion value. Then we can get the optimal number of clusters $k$. The correlation distance defined as one minus the sample correlation between points (treated as $Z_{i}$ ) is used to capture the closeness between individuals in $k$-means algorithm, such as,

$$
d_{i, m}=1-s_{i, m} .
$$

The $k$-means algorithm returns the cluster indices of each individual and the $k$ cluster centroid locations in a $k$-by-30 matrix. Each centroid is the component-wise mean of the points in that cluster. We denote the $k$ th cluster centroid locations as $P_{k}=$ $\left(P_{k, 1}, P_{k, 2}, \ldots, P_{k, 30}\right)$, where $P_{k, j}=\sum_{i \in \mathcal{C}_{k}} p_{i, j} / c_{k}$ and $j \in\{1,2, \ldots, 30\} . c_{k}$ is the number of individuals in cluster $\mathcal{C}_{k}$. We have $\sum_{j=1}^{30} P_{k, j}=1$, and $P_{k}$ is the position ratio profile of cluster $k$. 


\section{Results}

\subsection{Quantifying position ratio profile}

Individuals inside the virtual societies are involved in all kinds of social and economic activities. The theory of structural holes already tells us that the agent occupies special position in social network will have great influence on his economic performance [43]. Here, we empirically investigate the underlying connections between network structure and economic output for individuals in 248 virtual societies. Our data are game logs from a popular MMORPG in China (see data description in Section 2). For each society, we construct a dependence network of individuals, which only keeps the multiscale backbone of original friendship network (see construction of dependence network in Section 2). Figure 1 illustrates the dependence network from a virtual society and some basic statistics about the topological structure. One can see that there are lots of in-stars in dependence network, which is in accordance with the findings [4] that the in-degree distribution is much fatter than out-degree distribution. Such phenomena can be explained by that in virtual societies the individuals with high levels may play a relatively important role in friend lists of low level individuals, which is supported by the strong preference of connecting high level individuals for low level individuals [4].

For a given dependence network, we can estimate a position ratio profile for each individual (see quantifying position ratio profile in Section 2). Xie et al. revealed that the open motifs have higher occurrence frequency than close motifs [4], suggesting that the positions in the open motifs may have higher occurrence frequency than positions in close motifs. Figure 3(A) shows the position ratio profile for all individuals in dependence networks in Figure 1. There are more zeros in the position ratio profiles of individuals with low degrees than those with high degrees. More extremely, there are lots of individuals occupying only one specific position $(j=3,9,10,16,17$, and 18) of triadic motifs in dependence networks, which leads to $p_{i, j}=1$. The individuals with larger values of $p_{i, 3}, p_{i, 9}$, $p_{i, 16}$ in their position ratio profile may correspond to leaf nodes in dependence networks. Note that the occurrence frequency of motif (a) $\rightarrow$ (b) $\rightarrow$ (c) $\rightarrow$ (a) is 0 , resulting in that the position 21 is impossible to be observed.

\subsection{Similarity of position ratio profiles between individuals}

The triadic motifs are regarded as building blocks of complex networks [9], suggesting that the 30 positions in triadic motifs may contain important structural information of nodes in complex networks. Here we utilize the occurrence frequency of the 30 different positions in dependence networks to represent the network structure profile for each individual. Based on these profiles, we can assess the structural similarity between individuals, which allows us further to group individuals. In dependence networks, it is observed that some motifs, for example, (3) $\rightarrow$ (4) $\rightarrow$ (5), (9) $\rightarrow$ (10) $\leftarrow$ (9), (16) $\rightarrow$ (17) $\rightleftharpoons$ (18), appears more frequency than other motifs [4].

By ordering the individuals according to the rule that nodes with large similarity are close to each other, we plot the structural similarity in Figure 3(D). The color bar stands for the value of similarities $s_{i, m}$. One intriguing observation is that there is a block-diagonal structure, strongly indicating the function of grouping individuals for position ratio profiles. This inspires us to further classify the individuals into clusters by $k$-means algorithm (see classifying individuals based on their position ratio profile in Section 2). In Figure 3(A) and Figure 3(D), there are some classes which are made up of some special individuals, such 


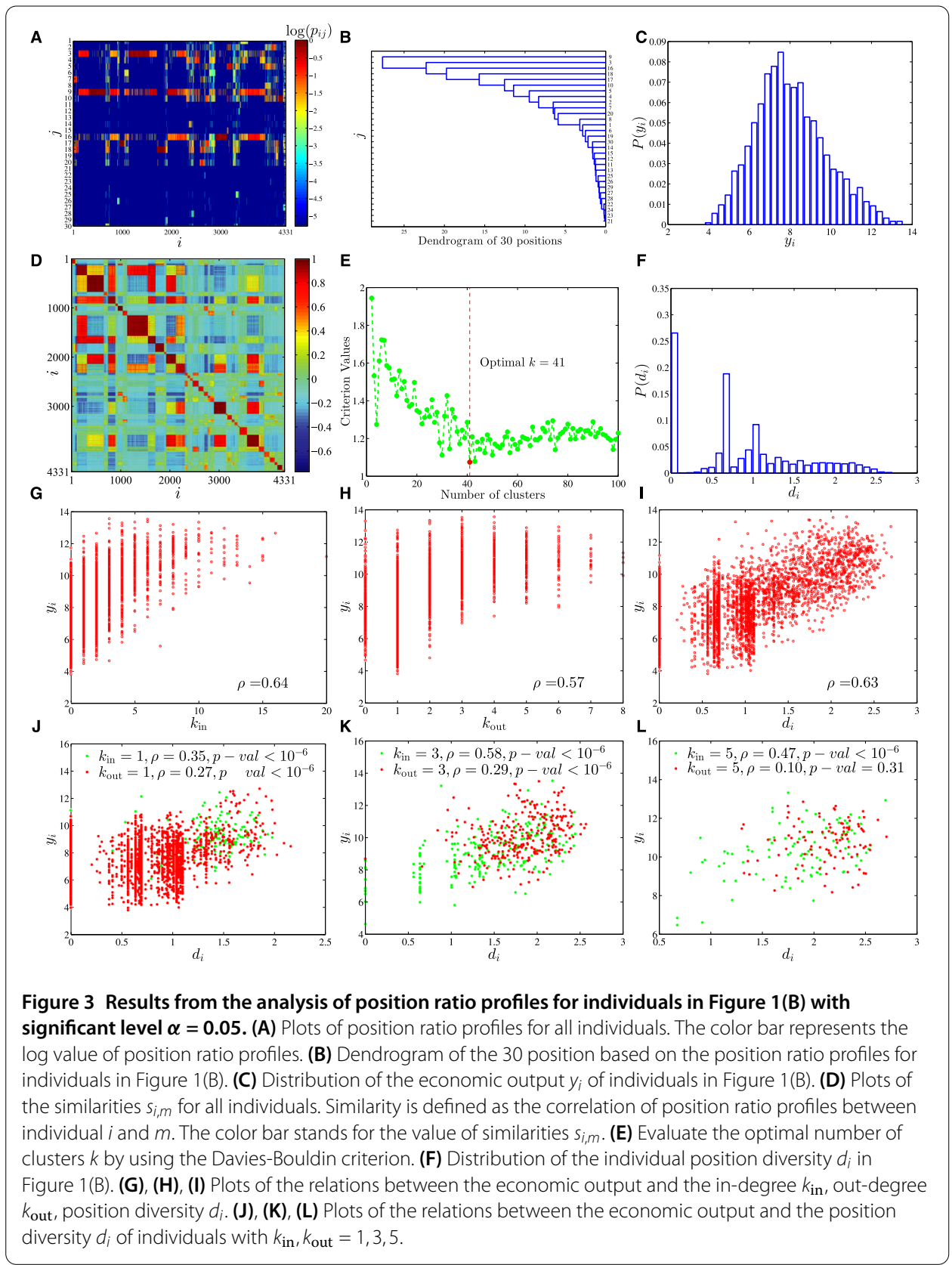

as individuals with $p_{i, 3}=1$ in motif (3) $\rightarrow$ (4) $\rightarrow$ (5), or individuals with $p_{i, 9}=1$ or $p_{i, 10}=1$ in motif (9) $\rightarrow$ (10) $\leftarrow$ (9), or individuals with $p_{i, 16}=1, p_{i, 17}=1$, or $p_{i, 18}=1$ in motif (16) $\rightarrow$ (17) $\rightleftharpoons$ (18).

The $k$-means algorithm is an iterative partitioning algorithm, which maximizes the similarity of the within-cluster. To evaluate the optimal number of clusters, we create a clustering evaluation object containing Davies-Bouldin index values in Figure 3(E). From the minimum criterion value, we can get the optimal number of clusters $k=41$. Based on the definition of position ratio profiles, there is a correlation among the 30 positions. From the dendrogram of the 30 position in Figure 3(B), we can find that a avatar at position 22 has a higher chance to be also in position 23 or 24 in the same motif. But there is no obvious classification among the 30 position. The block-diagonal structure cannot be explained by this relation among the 30 position. An alternative method to elucidate the 
position significant profiles is to calculate the difference of position ratio profiles between the dependence networks and the reference randomized dependence networks [9]. But this method is too computationally intensive for our 248 dependence networks and some of the networks' sizes are larger than fifty thousand.

\subsection{Relationship between position ratio profiles and economic outputs}

In social network, the individuals in key positions may have great impacts of network dynamics if some controlling strategies are applied on these key nodes. This leads to the conjecture that the position ratio profiles may have potential influence on the economic outputs for individuals. We estimate the economic output $y_{i}$ for each individual in a given world (see economic outputs of individuals in Section 2). To make these economic outputs comparable between different virtual worlds, we standardize the economic output within each society. The standardization of economic performance of individual $i$ is defined as output per capita, $Y_{i}=\left[y_{i}-\left\langle y_{i}\right\rangle\right] / \sigma\left(y_{i}\right)$, where $\left\langle y_{i}\right\rangle$ and $\sigma\left(y_{i}\right)$ are the mean and standard deviation of $y_{i}$ over all individuals in a given society. Therefore, the mean of economic performance of the class $\mathcal{C}_{k}$ can be estimated via $Y_{k}=\sum_{i \in \mathcal{C}_{k}} Y_{i} / c_{k}$, where $c_{k}$ is the number of individuals in class $\mathcal{C}_{k}$.

Motivated by social diversity [5], we define the individual position diversity $d_{i}$ as a function of Shannon entropy to quantify how individual appears in the 30 positions:

$$
d_{i}=-\sum_{j=1}^{30} p_{i, j} \ln p_{i, j} .
$$

Figure 3 shows the results from the analysis of position ratio profiles for individuals in Figure 1 with significant level $\alpha=0.05$. The position ratio profiles of 4,331 individuals are presented in Figure 3(A). To improve the visualization, the color bar represents the log value of position ratio profiles $p_{i, j}$. The position diversity $d_{i}=0$ means that the individual only occupy one triadic motif position. As shown in Figure 3(A), there are individuals with $p_{i, 3}=1$ in motif (3) $\rightarrow$ (4) $\rightarrow$ (5), $p_{i, 9}=1, p_{i, 10}=1$ in motif (9) $\rightarrow$ (10) $\leftarrow$ (9), $p_{i, 16}=1, p_{i, 17}=1$, and $p_{i, 18}=1$ in motif (16) $\rightarrow(17) \rightleftharpoons\left(18\right.$. Figure $3(\mathrm{C}),(\mathrm{F})$ show the economic output $y_{i}$ and the individual position diversity $d_{i}$ respectively. The individuals with position diversity $d_{i}=0$ account for a large part of avatars in the dependence network of a given society. Figure 3(L) shows the scatter plot of economic output $y_{i}$ and position diversity $d_{i}$. The correlation coefficient $\rho$ is 0.63 and $p$-value is less than $10^{-6}$, implying that the correlation between $y_{i}$ and $d_{i}$ is positive and highly significant. Our results indicate that the individuals who appear in more triadic motif positions have higher economic outputs. It is easy to find that more active players have more friends (more in-degree and out-degree) and appear in more triadic motif positions. Here we show the plots of the relation between the economic output $y_{i}$ and the individuals' in-degree $k_{\text {in }}$, out-degree $k_{\text {out }}$ in Figure $3(\mathrm{G}),(\mathrm{H})$ respectively. This agrees with Fuchs' result [44] that the output is correlated to both in- and out-degree. To avoid the impact of the in- and out-degree, we investigate the relation between the economic output $y_{i}$ and the position diversity $d_{i}$ of individuals with fixed $k_{\text {in }}, k_{\text {out }}=1,3,5$ in Figure 3(J), (K), (L). And we can get the same conclusion that the individual position diversity increases economic output of individuals with fixed $k_{\text {in }}$ or $k_{\text {out }}$. What is more, we also find that the economic output is susceptible to asymmetries between individuals' in- and out-degree in our dependence socioeconomic network. The individuals with high 
in-degree have higher economic outputs than with high out-degree. Because it is difficult to get the records and measure the activity of individuals accurately, we assume that the individuals with the same in- and out-degree have almost the same activity.

The $k$-means algorithm also gives cluster centroid locations denoted as $P_{k}=\left(P_{k, 1}, P_{k, 2}\right.$, $\ldots, P_{k, 30}$ ) for each class $\mathcal{C}_{k}$. Similar to equation (5), we define the cluster position diversity as $D_{k}=-\sum_{j=1}^{30} P_{k, j} \ln P_{k, j}$. In Figure 4(A)-(F), the clusters are sorted by the cluster position diversity $D_{k}$ in ascending order. Considering the impact of different significant level $\alpha$ on the relation between the economic output and position diversity, we analyze the dependence network from all the virtual society with different significant level $\alpha=0.01,0.05,0.1$. In Figure 4, the three columns from left to right correspond to the dependence networks with significant level $\alpha=0.01,0.05,0.1$ respectively. Figure 4(A), (B), (C) show the plots of the average economic output $Y$ of classes and Figure 4(D), (E), (F) illustrate the plots of the value of $P_{k}$ for different significant level $\alpha=0.01,0.05,0.1$ respectively. The clusters are sorted by the cluster position diversity $D_{k}$ in ascending order, so one can see that the economic output $Y$ increases with the cluster position diversity $D_{k}$. We can find that lots of clusters are comprised of individuals with $P_{k, 3}, P_{k, 9}, P_{k, 16}$ equalling to one. Although these individuals have the same individual position diversity $d_{i}=0$, their economic outputs could be different. For each dependence network with a given $\alpha$, we calculate the Davies-Bouldin index values to evaluate the optimal number of clusters and get the optimal number of clusters $k$. The distributions of the optimal number $k$ are shown in Figure 4(G), (H), (I). The average optimal number $k$ is between 50 and 60 .

To compare the relation coefficients between the economic output and the individuals' in-degree $k_{\text {in }}$, out-degree $k_{\text {out }}$, position diversity $d_{i}$, we denote the coefficients as $\rho_{k_{\text {in }}}, \rho_{k_{\text {out }}}$ and $\rho_{d_{i}}$ respectively and draw the distribution of the three kinds of coefficients for different significant level $\alpha$. When the significant levels $\alpha$ is smaller, there are greater difference between the three kinds of coefficients. At the same time, the asymmetries between individuals' in- and out-degree in our dependence socioeconomic network is obvious with the significant level $\alpha=0.01$.

\subsection{Economic output of individuals with the position diversity $d_{i}=0$}

Here, we conduct a comparison of the economic output of individuals with individual position diversity $d_{i}=0$ in the dependence networks from all the virtual society with significant level $\alpha=0.01,0.05,0.1$. The individual position diversity $d_{i}=0$ means that the individual appears only in one triadic motif position. We perform $t$-tests on pairs of economic output of the individuals at two position in the same motif. Figure 5 shows the box plots of economic output of individuals with position diversity $d_{i}=0$ for 30 unique positions $j \in\{1,2, \ldots, 30\}$ in 13 directed triadic motifs.

In Figure 5, the superscripts ***, **** stand that the relationship is acceptable at the significant level $0.05,0.001$ and 0.0001 respectively. For all the dependence networks with significant level $\alpha=0.01,0.05,0.1$, the results of the $t$-tests are similar.

In Figure 5, we perform $t$-tests on pairs of individual economic output of the individuals at two position in the dependence networks. It is worthy stressing that the mean economic output of individual at position 1 is greater than that of individuals within position 2 in motif (2) $\leftarrow$ (1) $\rightarrow$ (2). This relationship can be expressed as $1>2$ in Figure 5 . The mean economic output of individuals at position 4 and 5 are greater than that of individuals at position 3 in motif (3) $\rightarrow$ (4) $\rightarrow$ (5). These relationship can be expressed as $4>3$ and $5>3$ 


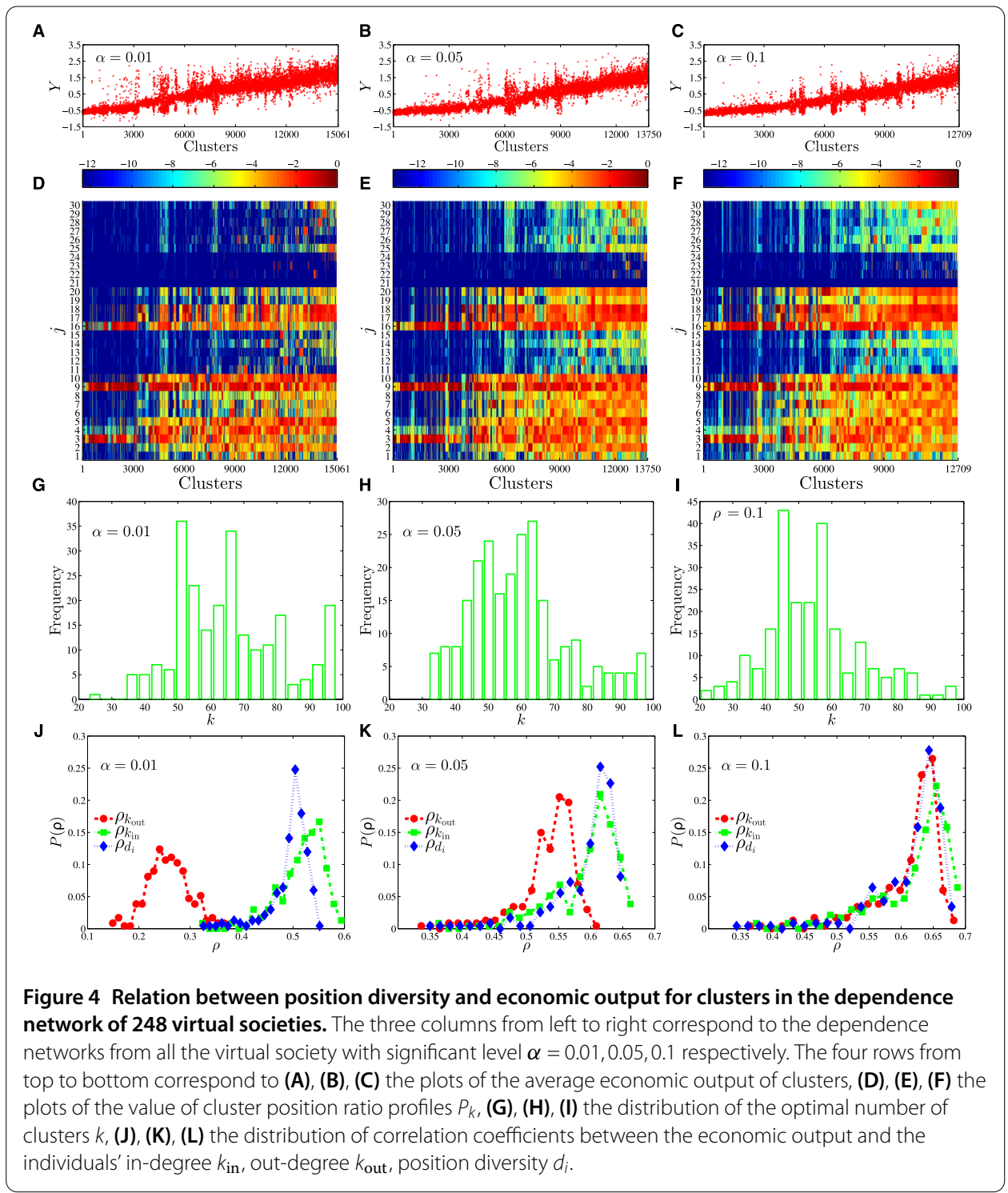

in Figure 5. The mean economic output of individuals at position 10 is greater than that of individuals at position 9 in motif (9) $\rightarrow$ (10) $\leftarrow$ (9). This relationship can be expressed as $10>9$ in Figure 5. The mean economic output of individuals at position 17 and 18 are greater than that of individuals at position 16 in motif (16) $\rightarrow(17) \rightleftharpoons(18)$. These relationship can be expressed as $17>16$ and $18>16$ in Figure 5 .

Comparing the output for individuals at different positions in the dependence networks with significant level $\alpha=0.05$, the 30 positions can be classified into two groups. The first group contains the positions: (3), (16), (9), (19), (18), (4), (17), (5), (7), (20), (8), (13), (2), (26). The second group contains positions: (25), (23), (12), (24), (11), (19), (6), (15), (29), (22), (1), (27), (32), (14), (28). All the positions are sorted by means of individual economic output in ascending order. The mean economic output of individuals in the first group is less than 0 , while in the second group the mean output is greater than 0 . Such differences could result from that most of positions of the first group are in open motifs while most of positions in second group belong to close motifs. 

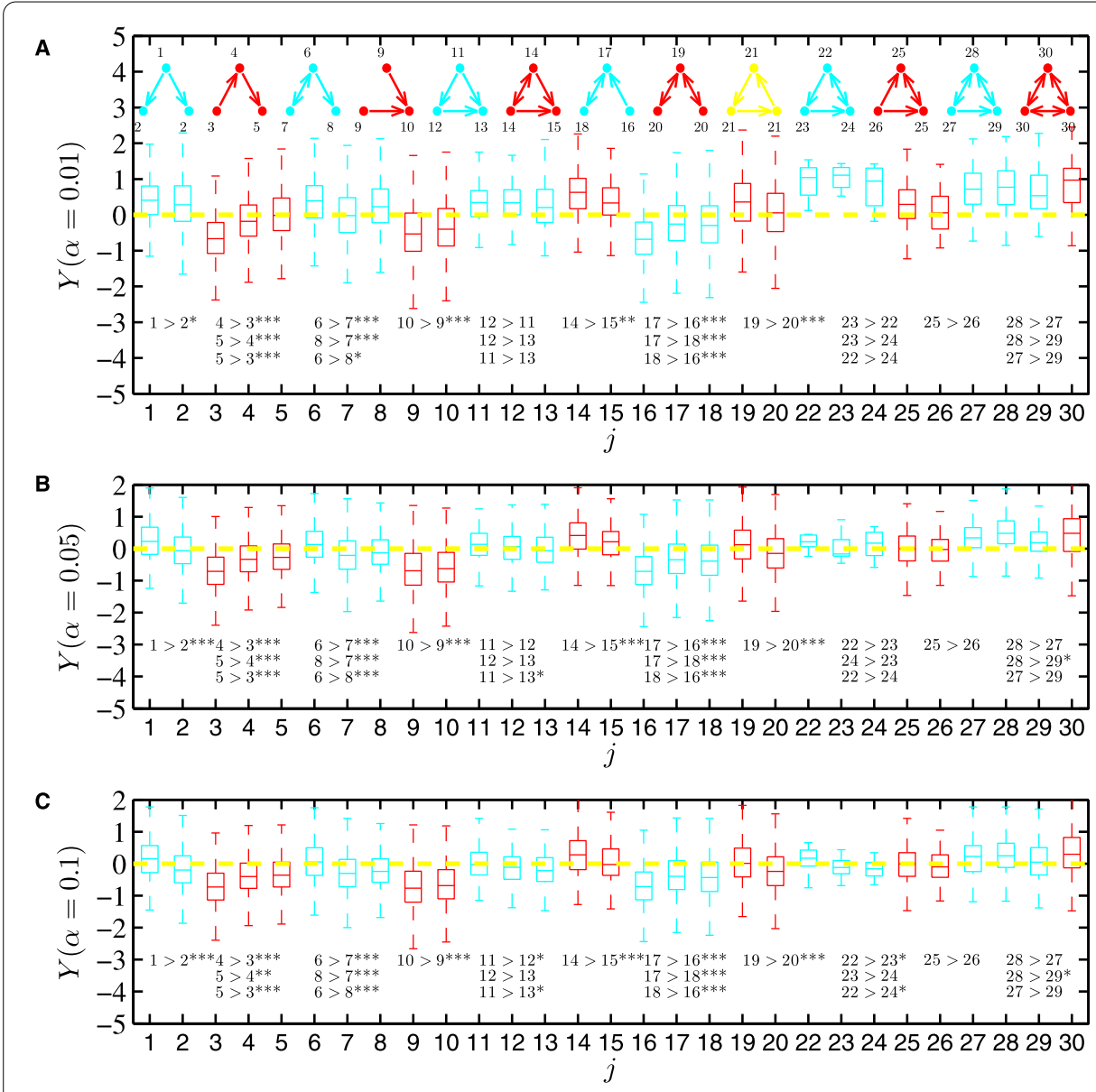

Figure 5 Different economic output of individuals with the same position diversity $d_{i}=0$ in the dependence networks from all the virtual societies with significant level $\boldsymbol{\alpha}=0.01,0.05,0.1$. Boxplots of economic output of individuals with position diversity $d_{i}=0$ for 30 unique positions $j \in\{1,2, \ldots, 30\}$ in 13 directed triadic motifs. The colors are only visually easier to distinguish the positions in the two adjacent motifs. There is no practical meaning. We perform $t$-tests on pairs of economic output of the individuals at two position in the same motif. The mean economic output of individual at position 1 is greater than that of individuals within position 2 in motif (2) $\leftarrow$ (1) $\rightarrow$ (2). This relationship can be expressed as $1>2$. The superscript $*^{*},{ }^{* *},{ }^{* *}$ stand that the relationship is acceptable at the significant level $0.05,0.001,0.0001$ respectively.

\section{Discussion}

In this paper we analyzed the relationship between friendship structure and economic output for individuals in a massively multiplayer online role-playing game. We found that the individual position diversity of individuals is positively correlated with their economic output and social status. We have developed a new approach to study structural similarity of individuals in networks and classify individuals into different clusters based on their position ratio profiles. It is found that the economic output of leaf nodes is significantly lower than the nodes at the other triadic motif positions. The individual position diversity positively correlates with the economic output. Our results are in consistent with the results from the physical society $[3,13]$. Furthermore, Eagle et al. found that the diversity of individual relationships within a community strongly correlates with the economic development of the community [5]. In a recent analysis, Xie et al. found the degree of skill complementarity is positively correlated with their output [40]. 
Much evidence shows that the behaviour of individuals in virtual society is representative in many aspects of human behaviour in physical society. This is rational because individuals' decision process is determined by players that are real human being. The invisible hand operates not only in modern societies but also in ancient societies, not only in real societies but also in virtual societies. Yee et al. argued that online environments such as MMORPGs could potentially be unique research platforms for the social sciences and clinical therapy, but it is crucial to firstly establish that social behavior and norms in virtual environments are comparable to those in the physical world [45]. To investigate the relation between friendship (or socioeconomic) networks in virtual and real worlds, Grabowski and Kruszewska conducted a survey among the players of an online game and construct the off-line network [39]. They showed that the structure of the friendship network in virtual world was very similar to the structure of different social networks in real world.

We believe that our interdisciplinary work represents significant scientific evidence for understanding the behaviour of social systems. It involves topics that range from social science, network science, and economics to human dynamics. It also enriches our understanding on the formation of socio-economic networks and proposes a new method to classify nodes of complex networks for understanding of people's economic behaviour from the big data of massive players. Our work sheds new light on the scientific research utility of virtual worlds for studying human behaviour in complex socio-economic systems.

Acknowledgements

We are grateful to Qun-Zhao Tan for providing data and discussions.

\section{Funding}

This work was supported by the National Natural Science Foundation of China [11505063, 11375064 and 11605062]; the Shanghai Chenguang Program [15CG29]; the Ph.D. Programs Foundation of Ministry of Education of China [20120074120028]; the China Postdoctoral Science Foundation [2016M600291] and the Fundamental Research Funds for the Central Universities [222201718006].

\section{Abbreviations}

MMORPG: Massively Multiplayer Online Role-Playing Game

Availability of data and materials

We use a huge database recorded from 124 servers of a popular Massively Multiplayer Online Role-Playing Game operated by a leading online game company in China. And we signed a confidentiality agreement, so the data will not be shared.

\section{Competing interests}

The authors declare that they have no competing interests.

Authors' contributions

W-JX, Z-QJ, W-XZ conceived the research. W-JX and W-XZ designed the analyses. W-JX, Y-HY, M-XL and Z-QJ compiled the data. W-JX, Y-HY, M-XL, Z-QJ and W-XZ conducted the analyses. All authors discussed the results. W-JX, Z-QJ and W-XZ wrote the paper. All authors read and approved the final manuscript.

\section{Author details}

${ }^{1}$ School of Business, East China University of Science and Technology, Meilong Road, 200237, Shanghai, China. ${ }^{2}$ Research Center for Econophysics, East China University of Science and Technology, Meilong Road, Shanghai, China. ${ }^{3}$ Research Institute of Sports Economics, East China University of Science and Technology, Meilong Road, Shanghai, China.

${ }^{4}$ Department of Mathematics, East China University of Science and Technology, Meilong Road, Shanghai, China.

\section{Publisher's Note}

Springer Nature remains neutral with regard to jurisdictional claims in published maps and institutional affiliations. 


\section{References}

1. Wang P, González MC, Hidalgo CA, Barabási A-L (2009) Understanding the spreading patterns of mobile phone viruses. Science 324:1071-1076. doi:10.1126/science.1167053

2. Newman MEJ (2001) The structure of scientific collaboration networks. Proc Natl Acad Sci USA 98:404-409

3. Bhattacharya H, Dugar S (2014) Partnership formation: the role of social status. Manag Sci 60(5):1130-1147. doi:10.1287/mnsc.2013.1818

4. Xie W-J, Li M-X, Jiang Z-Q, Zhou W-X (2014) Triadic motifs in the dependence networks of virtual societies. Sci Rep 4:5244. doi:10.1038/srep05244

5. Eagle N, Macy M, Claxton R (2010) Network diversity and economic development. Science 328(5981):1029-1031. doi:10.1126/science.1186605

6. Bettencourt LMA, Samaniego H, Youn H (2014) Professional diversity and the productivity of cities. Sci Rep 4:5393. doi:10.1038/srep05393

7. Ortman SG, Cabaniss AHF, Sturm JO, Bettencourt LMA (2015) Settlement scaling and increasing returns in an ancient society. Sci Adv 1(1):1400066. doi:10.1126/sciadv.1400066

8. Milo R, Itzkovitz S, Kashtan N, Levitt R, Shen-Orr S, Ayzenshtat I, Sheffer M, Alon U (2004) Superfamilies of evolved and designed networks. Science 303:1538-1542. doi:10.1126/science.1089167

9. Milo R, Shen-Orr S, Itzkovitz S, Kashtan N, Chklovskii D, Alon U (2002) Network motifs: simple building blocks of complex networks. Science 298:824-827. doi:10.1126/science.298.5594.824

10. Milo R, Kashtan N, Itzkovitz S, Newman MEJ, Alon U (2004) Uniform generation of random graphs with arbitrary degree sequences. http://arxiv.org/abs/cond-mat/0312028

11. Kovanen L, Kaski K, Kertész J, Saramäki J (2013) Temporal motifs reveal homophily, gender-specific patterns, and group talk in call sequences. Proc Natl Acad Sci USA 110(45):18070-18075. doi:10.1073/pnas.1307941110

12. Klimek P, Thurner S (2013) Triadic closure dynamics drives scaling laws in social multiplex networks. New J Phys 15(6):063008. doi:10.1088/1367-2630/15/6/063008

13. Ball B, Newman MEJ (2013) Friendship networks and social status. Netw Sci 1:16-30. doi:10.1017/nws.2012.4

14. Palla G, Barabási A-L, Vicsek T (2007) Quantifying social group evolution. Nature 446:664-667. doi:10.1038/nature05670

15. Onnela J-P, Saramäki J, Hyvönen J, Szabó G, Lazer D, Kaski K, Kertész J, Barabási A-L (2007) Structure and tie strengths in mobile communication networks. Proc Natl Acad Sci USA 104:7332-7336. doi:10.1073/pnas.0610245104

16. Kumpula JM, Onnela J-P, Saramäki J, Kaski K, Kertész J (2007) Emergence of communities in weighted networks. Phys Rev Lett 99:228701. doi:10.1103/PhysRevLett.99.228701

17. Eagle N, Pentland A, Lazer D (2009) Inferring friendship network structure by using mobile phone data. Proc Natl Acad Sci USA 106(106):15274-15278. doi:10.1073/pnas.0900282106

18. Jo H-H, Pan RK, Kaski K (2011) Emergence of bursts and communities in evolving weighted networks. PLoS ONE 6:22687. doi:10.1371/journal.pone.0022687

19. Jiang Z-Q, Xie W-J, Li M-X, Podobnik B, Zhou W-X, Stanley HE (2013) Calling patterns in human communication dynamics. Proc Natl Acad Sci USA 110(5):1600-1605. doi:10.1073/pnas.1220433110

20. Currarini S, Jackson MO, Pin P (2009) An economic model of friendship: homophily, minorities and segregation. Econometrica 77(4):1003-1045. doi:10.3982/ECTA7528

21. Currarini S, Jackson MO, Pin P (2010) Identifying the roles of race-based choice and chance in high school friendship network formation. Proc Natl Acad Sci USA 107(11):4857-4861. doi:10.1073/pnas.0911793107

22. Uzzi B (1996) The sources and consequences of embeddedness for the economic performance of organizations: the network effect. Am Sociol Rev 61(4):674-698

23. Guimera R, Uzzi B, Spiro J, Amaral L-A-N (2014) Team assembly mechanisms determine collaboration network structure and team performance. Science 308(5277):697-702. doi:10.1126/science.1106340

24. Cantner U, Joel K (2011) Network position, absorptive capacity and firm success. IUP J Know Manag 9(1): 37-56

25. Garas A, Tomasello M-V, Schweitzer F (2014) Newcomers vs. incumbents: How firms select their partners for R\&D collaborations. ArXiv preprint. arXiv:1403.3298

26. Jiang Z-Q, Zhou W-X, Tan Q-Z (2009) Online-offline activities and game-playing behaviors of avatars in a massive multiplayer online role-playing game. Europhys Lett 88(4):48007. doi:10.1209/0295-5075/88/48007

27. Jiang Z-Q, Ren F, Gu G-F, Tan Q-Z, Zhou W-X (2010) Statistical properties of online avatar numbers in a massive multiplayer online role-playing game. Physica A 389:807-814. doi:10.1016/j.physa.2009.10.028

28. Thurner S, Szell M, Sinatra R (2012) Emergence of good conduct, scaling and Zipf laws in human behavioral sequences in an online world. PLoS ONE 7(1):29796. doi:10.1371/journal.pone.0029796

29. Szell M, Sinatra R, Petri G, Thurner S, Latora V (2012) Understanding mobility in a social petri dish. Sci Rep 2:457. doi:10.1038/srep00457

30. Szell M, Thurner S (2012) Social dynamics in a large-scale online game. Adv Complex Syst 15(6):1250064 doi:10.1142/S0219525912500646

31. Bainbridge WS (2007) The scientific research potential of virtual worlds. Science 317(5837):472-476 doi:10.1126/science.1146930

32. Papagiannidis S, Bourlakis M, Li F (2008) Making real money in virtual worlds: MMORPGs and emerging business opportunities, challenges and ethical implications in metaverses. Tech Forcast Soc Change 75:610-622. doi:10.1016/j.techfore.2007.04.007

33. Williams D (2010) The mapping principle, and a research framework for virtual worlds. Commun Theory 20(4):451-470. doi:10.1111/j.1468-2885.2010.01371x

34. Chesney T, Chuah S-H, Hoffmann R (2009) Virtual world experimentation: an exploratory study. J Econ Behav Organ 72:618-635. doi:10.1016/j.jebo.2009.05.026

35. Szell M, Lambiotte R, Thurner S (2010) Multirelational organization of large-scale social networks in an online world Proc Natl Acad Sci USA 107(31):13636-13641. doi:10.1073/pnas.1004008107

36. Szell M, Thurner S (2010) Measuring social dynamics in a massive multiplayer online game. Soc Netw 32(4):313-329. doi:10.1016/j.socnet.2010.06.001

37. Szell M, Thurner S (2013) How women organize social networks different from men. Sci Rep 3:1214. doi:10.1038/srep01214 
38. Grabowski A, Kosiński R (2008) The SIRS model of epidemic spreading in virtual society. Acta Phys Pol A 114:589-596

39. Grabowski A, Kruszewska N (2007) Experimental study of the structure of a social network and human dynamics in a virtual society. Int J Mod Phys C 18:1527-1535. doi:10.1142/S0129183107011480

40. Xie W-J, Li M-X, Jiang Z-Q, Tan Q-Z, Podobnik B, Zhou W-X, Stanley HE (2016) Skill complementarity enhances heterophily in collaboration networks. Sci Rep 6:18727. doi:10.1038/srep18727

41. Serrano MÁ, Boguñá M, Vespignani A (2009) Extracting the multiscale backbone of complex weighted networks. Proc Natl Acad Sci USA 106:6483-6488. doi:10.1073/pnas.0808904106

42. Stouffer DB, Sales-Pardo M, Sirer MI, Bascompte J (2012) Evolutionary conservation of species' roles in food webs. Science 335:1489-1492. doi:10.1126/science.1216556

43. Burt RS (2009) Structural holes: the social structure of competition. Harvard University Press, Cambridge

44. Fucha B, Thurner S (2014) Behavioral and network origins of wealth inequality: insights from a virtual world. PLoS ONE 9(8):103503. doi:10.1371/journal.pone.0103503

45. Yee N, Bailenson JN, Urbanek M, Chang F, Merget D (2007) The unbearable likeness of being digital: the persistence of nonverbal social norms in online virtual environments. CyberPsychol Behav 10(1):115-121. doi:10.1089/cpb.2006.9984

\section{Submit your manuscript to a SpringerOpen ${ }^{\circ}$} journal and benefit from:

- Convenient online submission

Rigorous peer review

- Open access: articles freely available online

- High visibility within the field

- Retaining the copyright to your article

Submit your next manuscript at $\gg$ springeropen.com 